\title{
Estudo da adaptação antropométrica de mobiliário residencial em madeira maciça produzido na cidade de Manaus
}

\author{
Alencar, Larissa Albuquerque ${ }^{a}$; Pacheco, Karla Mazarelo Maciel ${ }^{b}$ \& Vieira, Mirella Sousa ${ }^{c}$ \\ ${ }^{a}$ Mestre em Ciências Florestais e Ambientais - Departamento de Design e Expressão Gráfica, Universidade Federal \\ do Amazonas, Brasil - larissa_alencar@ufam.edu.br, \\ ${ }^{\mathrm{b}}$ Doutora em Design - Departamento de Design e Expressão Gráfica, Universidade Federal do Amazonas, Brasil - \\ karlamazarelo@hotmail.com, \\ ${ }^{c}$ Mestre em Ciências Florestais e Ambientais - Departamento de Design, Martha Falcão DeVry, Brazil - \\ mirellasou@gmail.com,
}

\begin{abstract}
Resumo
Este artigo apresenta um breve estudo sobre a adaptação antropométrica de mobiliário de madeira maciça, produzido e comercializado na cidade de Manaus. Neste, buscou-se verificar se as medidas aplicadas à produção de mesas e cadeiras (sem ajustes) estão em conformidade com as normas e padrões técnicos vigentes. Para tanto, realizou-se: levantamento bibliográfico, abordando a situação do setor moveleiro no Amazonas, ergonomia e antropometria, buscando aprofundar os conhecimentos acerca da temática estudada, conhecendo-se as principais limitações e potenciais para investimento na melhoria da qualidade do mobiliário de madeira maciça sem ajustes. Organizou-se os dados pesquisados para a geração de duas tabelas, com base nas dimensões dos seguimentos corpóreos humanos de usuários extremos (mulher percentil 5\% e homem percentil 95\%), apresentando recomendações de diversos autores de ergonomia, , viabilizando seu uso como parâmetro para o dimensionamento de mesas e cadeiras. Além de pesquisa de campo, para anotação das medidas e comparação posterior com as dimensões das tabelas propostas; e cálculos de estatística descritiva, e inferencial, com o propósito de facilitar a apreciação acerca da validade dos dados coletados por meio da aplicação do teste de $t$ de Student. Como resultado, observou-se que o mobiliário em questão não é produzido com base em recomendações ergonômicas elou quaisquer tipo de estudo relacionado, uma vez que as medidas analisadas encontravam-se em conformidade com as referencias adequadas e outras eram simplesmente ignoradas. Tornando os produtos inadequados, face ao desconforto gerado, quando utilizados por longos períodos, principalmente se comparados com móveis industrializados, tendo em vista que estes apresentam as mesmas condições de dimensionamento com menor preço de mercado.
\end{abstract}

Keywords: Design, ergonomia, antropometria, mobiliário, madeira maciça. 


\begin{abstract}
This article presents a brief study about the anthropometric adaptation of solid wood furniture, manufactured and commercialized in the city of Manaus. It searches to verify if the measures used to the production of tables and chairs (without adjustments) are in conformity with the current standard norms and patterns. Therefore, it were made: bibliographic collection, where topics referred to the situation of the furniture sector in Amazonas were approached, ergonomics and anthropometric and human body measures, in an effort to deepen the findings about the studied theme, by means of the knowledge of the main limitations and potentials to the investment in the improvement of the quality of the solid wood furniture without adjustments. In this section, there were an organization of the researched data to the creation of two tables, based on the dimensions of human body segments of extreme users (woman percentile 5\% and man percentile 95\%), presented by Felisberto and Paschoarelli (2000), following as well the recommendations of various authors of ergonomics, between them: Quaresma (2011), Kroemer and Grandjan (2005), Dul and Weerdemester (2004) and Moraes and Pequini (2000), enabling its usage as a parameter to the sizing of tables and chairs; field research, where there was the collection of data through search form for annotation of the measures obtained in loco on the carpentry shops, to be afterwards compared to the dimensions of the suggested tables; calculations of descriptive statistic (total amplitude, standard deviation and coefficient of variation), since they allow to appreciate the total number of values found in a class and map the differentiation among those obtained and recommended, and inferential, with the intension of facilitate the appreciation on the validity of the collected data by means of the implementation of the $t$ Test of Student. With the obtained result, it was observed that the furniture in question is not produced based on ergonomic recommendations and/or any type of related study, as the measures analyzed were not found in conformity with the recommendations of the tables generated and the others were merely ignored. This fact ends up turning the products less attractive, in face of the discomfort created, when utilized by long periods of time, especially if compared to industrialized furniture, taking into consideration that those ones present the same condition of sizing with less Market price.
\end{abstract}

Keywords: Design, ergonomics, anthropometry, furniture, solid wood.

\title{
1. Introdução
}

As movelarias de Manaus possuem potencial para ganhar maior projeção no mercado local, pois os produtos de madeira maciça são mais resistentes e preferidos para decorar espaços com beleza e estilo. Entretanto, a Superintendência da Zona Franca de Manaus - SUFRAMA (2003) revelou em um estudo, que marcenarias locais possuem layout inadequado à produção, com equipamentos obsoletos e ausência de pessoal qualificado, o que compromete significativamente a qualidade dos móveis em madeira maciça produzidos localmente. 
Sabendo-se que o mercado atual prima pela eficiência produtiva e preço baixo do produto, tem-se buscado alternativas de design diferenciado e estratégias comerciais como formas de atender as necessidades dos consumidores. Para Souza et al. (2010) é cada vez maior a procura por produtos de melhor qualidade, que propiciem segurança e conforto durante o uso, sinalizando a importância da ergonomia na indústria moveleira como um fator de grande competitividade. Assim, o mobiliário residencial também deve ser projetado de modo a proporcionar conforto durante os mais diversos tipos de atividades, pois é fundamental que as pessoas adotem posturas corretas, evitando-se dores lombares e musculares (ombros, pescoço, nádegas, pernas e braços).

Nesse contexto, projetar móveis observando dimensões que respeitem os limites e esforços físicos do ser humano garante além do conforto, saúde e bem-estar. E tratando-se de mobiliário de madeira maciça e sem ajustes, os que merecem maior atenção em relação ao dimensionamento são mesas e cadeiras, objetos deste estudo, pois o dimensionamento incorreto desse mobiliário implica em prejuízos pela postura incorreta que se possa adotar, o que segundo Kroemer e Grandjean (2005), pode provocar anormalidades permanentes à coluna.

Souza et al. (2010) afimam, que ainda não existem normas técnicas direcionadas para mobiliário de uso residencial no Brasil, dificultando a elaboração de projetos de qualidade, facilitando a concorrência injusta com móveis de má qualidade disponíveis no mercado. Embora, tenha-se conhecimento de estudos realizados por Felisberto e Paschoarelli (2000) que apresentam valores tabelados dos segmentos corpóreos para a população brasileira. Estes, se usados corretamente, contribuem para a melhora na qualidade dos produtos produzidos e comercializados, proporcionando melhores condições de conforto e segurança à população.

Assim, esta pesquisa buscou verificar se os produtos de madeira maciça produzidos e comercializados na cidade de Manaus selecionados para este estudo, encontram suas medidas em conformidade com àquelas propostas pelos diversos autores de ergonomia conhecidos na atualidade, os quais foram organizados em duas tabelas geradas também como resultado deste estudo.

\section{Referencial teórico}

\subsection{O setor moveleiro no Amazonas}

Em conformidade com dados fornecidos pelo SEBRAE (Serviço Brasileiro de Apoio as Micro e Pequenas Empresas) em 2010, foram identificadas 456 movelarias nos municípios do Amazonas, e segundo informações da diretoria do mesmo orgão, deste total apenas 147 movelarias encontram-se atuantes na cidade de Manaus.

Sabe-se que o setor moveleiro apresenta uma série de problemas, e encontra em um estágio atrasado, visto que $80 \%$ dos estabelecimentos apresentam layout inadequado, entre outras dificuldades. Além disso, $84 \%$ dos empreendimentos trabalham em sistema de produção "sob encomenda" e somente $16 \%$ produzem em série (SUFRAMA, 2003), entre outros problemas frequentes destacam-se:

- Indefinição da situação fundiária;

- Perdas no transporte, principalmente quando realizado por via fluvial;

- Veículos inadequados para o transporte de madeira; 
- Ausência das técnicas de exploração e manejo adequados às espécies florestais, pré-requisito fundamental para obtenção da certificação ambiental e, consequentemente, garantia de venda no mercado internacional;

- Falta de visão empresarial nos aspectos administrativos, produtivos e mercadológicos por parte dos empresários atuantes no ramo;

- Ineficiência ou ausência de processo de secagem e/ou preservação da madeira;

- Baixo rendimento e deficiência quanto à qualidade do produto (por equipamentos obsoletos, falta de manutenção preventiva e mão-de-obra qualificada);

- Inobservância ou desconhecimento da legislação ambiental.

Além dos aspectos supracitados, deve-se salientar a ausência de preocupações relacionadas ao uso de normas e outras referências técnicas para determinação das dimensões dos mobiliários, principalmente no que diz respeito à utilização adequada das medidas antropométricas do público consumidor.

\subsection{Ergonomia aplicada à mobiliário sem ajustes}

A antropometria é o conjunto de medidas do corpo humano necessárias ao processo projetual de espaços, mobiliários e equipamentos, incluindo-se variáveis pertinentes à faixa etária, sexo, raça e, inclusive, grupos ocupacionais, conforme afirma Bittencourt (2011). Tais referências de variação nas dimensões humanas consideram-se de extrema importância, considerando as diferenças representativas a partir dos extremos de qualquer população.

Contudo, tratando-se de projetos de mobiliários, deve-se levar em conta a natureza da atividade a ser realizada, uma vez que as posturas naturais do corpo - posturas do tronco, braço e pernas que não envolvam trabalho estático - e movimentos naturais são condições necessárias para um trabalho eficiente, sendo imprescindível a adaptação do local de trabalho às medidas do corpo e à mobilidade do operador (KROEMER E GRANDJEAN, 2005).

Estas, também aplicam-se na construção de mobiliário residencial, pois os mesmos, assim como a mobília de postos de trabalho, devem ser projetados de modo a proporcionar conforto e segurança ao usuário durante sua utilização, diminuindo o aparecimento de lesões.

\subsubsection{Variáveis humanas aplicadas ao projeto de mobiliário}

Para o projeto de cadeiras, Panero e Zelnik (2003), consideram como uma das principais dificuldades em seu projeto o fato de que a atividade de sentar é dinâmica, ou seja, os indivíduos encontram-se em constante movimento pela adoção de diversas posturas, tratando-se da alternância entre contração e extensão muscular por meio de tensão e relaxamento dos músculos, conforme citam Kroemer e Grandjean (2005).

Fialho et al. (2007) afirmam, que existem poucas publicações a respeito de dados antropométricos dinâmicos e funcionais da população brasileira, dificultando a realização de projetos adaptados à população.

Contudo, um estudo intitulado "Modelos humanos em escala para dimensionamento ergonômico preliminar de postos de trabalho" proposto por Felisberto e Paschoarelli (2000) aponta medidas para o correto dimensionamento de mobiliário em geral, que podem ser utilizados para adequação do mobiliário residencial, pois apresenta um parâmetro antropométrico (medidas dos segmentos corpóreos humanos), tratado estatisticamente, obtido por meio de dados de diversas fontes de antropometria (tabelas de 
diversos autores e entidades competentes). Logo, a utilização deste estudo tende a contribuir para adequação do mobiliário residencial.

\subsubsection{Variações nas dimensões do corpo, faixas de projeto e percentis}

Para Kroemer e Grandjean (2005) a grande variabilidade nas medidas corpóreas entre indivíduos é um fator agravante na qualidade do mobiliário produzido na atualidade, considerando que não se deve projetar buscando atender a "pessoa média", e sim as pessoas mais altas (acomodar as pernas sob a mesa) ou as pessoas mais baixas (alcançar dada altura).

Atendendo, portanto aos usuários maiores e menores no projeto de postos de trabalho e de móveis, pois, geralmente, não é possível projetar o espaço de trabalho para atender as pessoas de dimensões extremas (muito grandes e muito pequenas), sendo necessário que se satisfaça às necessidades da maioria da população.

O uso de tabelas antropométricas pode ser entendido como referencial, sem, contudo, ser recomendado como prática indiferenciada, conforme afirma Bittencourt (2011), uma vez que existem variações dimensionais, tanto para homens quanto mulheres.

Assim, para que se possa projetar produtos para uma determinada população, torna-se necessário conhecer o conceito de percentil (p) que é definido como "unidade estatística (1 de 100) de uma distribuição normal da população" (BOUERI, 1991). Para Quaresma (2011) os percentis mais frequentemente utilizados são o $5 \%$, que apresentam as menores dimensões de uma população, e o $95 \%$ que representa maiores dimensões de uma população. Estes dois, em uma distribuição populacional normal, correspondem às medidas extremas de $90 \%$ de uma população.

Deste modo, caso decida-se projetar para o percentil 90\% central de uma população, deve-se excluir o $5 \%$ menor (mulheres com dimensões muito menores) e o $5 \%$ maior (homens dimensões muito maiores), uma vez que esses valores percentuais representam apenas $10 \%$ (5\% mulheres e $5 \%$ homens) de toda a população (KROEMER e GRANDJEAN, 2005).

Embora não existam normas específicas para dimensionamento de mobiliário residencial, conforme afirmam Souza et al. (2010), os dados utilizados para postos de trabalho lhe são úteis. Assim, optou-se por utilizar a tabela de segmentos corpóreos humanos proposta por Felisberto e Paschoarelli (2000).

A Figura 1, a seguir, identifica as medidas antropométricas tabeladas que serão utilizadas ao longo deste estudo para análise do correto dimensionamento do mobiliário. 

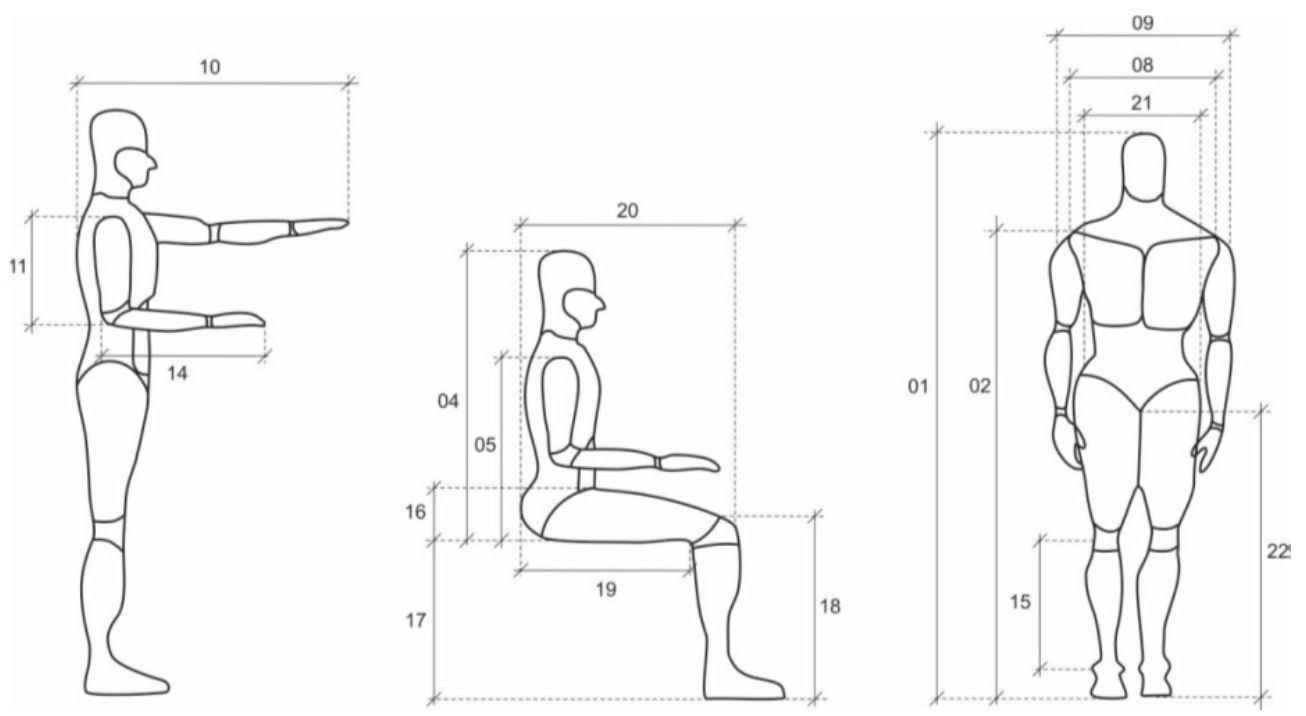

Fig. 30 Representação bidimensional das 29 variáveis antropométricas. Fonte: Felisberto e Paschoarelli (2000).

\section{Metodologia}

Para este trabalho, utilizou-se a pesquisa de campo, orientada por Marconi e Lakatos (2011), realizando as três etapas recomendadas: bibliográfica, coleta de dados e registro de dados coletados. Para a observação de campo, realizado no setor moveleiro do Amazonas, teve-se como universo de pesquisa 19 estabelecimentos, aplicando-se o método quantitativo-descritivo de verificação de hipótese, delineando e analisando as características dos fenômenos comprovados por controle estatístico. O estudo foi estruturado com base na ergonomia, direcionada aos aspectos antropométricos e medidas do corpo humano, tendo como referência os estudos orientados por Felisberto e Paschoarelli (2000) e complementados por Dul e Weedemester (2004), Quaresma (2011) e Moraes e Pequini (2000). Dos quais, foram geradas duas tabelas para experimento de aplicação das medidas para o desenvolvimento de mesas (34) e cadeiras (22) de madeira maciça. A partir dos dados gerados, pôs-se em prática uma análise comparativa com a realidade das medidas encontradas no mercado para esses produtos, a qual teve como suporte a estatística descritiva e inferencial. As observações registradas na referida fase, possibilitou verificar nos produtos analisados a existencia de variáveis compatíveis e não compatíveis ao seu processo de fabricação.

\section{Desenvolvimento}

A hipótese verificada partiu da premissa de que os marceneiros locais não utilizavam medidas antropométricas consideradas adequadas para o dimensionamento de mobiliário para a população local, não fazendo uso de nenhum padrão dimensional normatizado ou baseado em dados antropométricos. Para sua averiguação, elaborou-se um formulário de coleta de dados, possibilitando o registro das várias dimensões encontradas durante visita aos estabelecimentos apontados pelo SEBRAE/AM (CERVO, DA SILVA e BERVIAN, 2007).

A seleção da amostra de estabelecimentos a serem visitados para coleta de dados deu-se de acordo com a equação 1 a seguir: 


$$
n_{0}=\frac{\left(z_{\alpha / 2}\right)^{2} \cdot p(1-p)}{(e)^{2}}
$$

Aplicando-se a equação de amostragem proporcional (Equação 1), com erro tolerável igual a $8 \%(0,08)$ e intervalo de confiança de $95 \%(z=1,96)$, levando-se em conta a proporção de $10 \%(p=0,10)$ das movelarias, obteve-se a amostra inicial de 54 movelarias.

$$
\mathrm{n}=\frac{\mathrm{n}_{0}}{1+\frac{\mathrm{n}_{0}}{\mathrm{~N}}}
$$

Encontrado o valor da amostra inicial $\left(\mathrm{n}_{0}\right)$, tornou-se possível calcular o valor estimado da amostra (n), Equação 2, obtendo-se uma amostra total de 40 estabelecimentos.

A técnica de amostragem utilizada foi estratificada propocional, permitindo que se obtivesse um número de movelarias a serem visistadas em cada zona da cidade, partindo-se de uma população total contida nos estratos. Os dados referentes ao cálculo das amostras (n), proporção (p) e universo (N) de pesquisa encontram-se na Tabela 1.

Para sua aplicação, definiu-se como população, os estabelecimentos que comercializam mobiliário de madeira na cidade de Manaus, que conforme dados fornecidos pela diretoria do SEBRAE/AM (2010), era de aproximadamente 147 movelarias distribuídas pelas seis zonas da cidade. Do total da amostra estimada obtida, ressalta-se que foram incluídas apenas movelarias que trabalhavam com comercialização de mesas e cadeiras de madeira, excluindo-se as que não trabalhavam com a matéria-prima e mobiliário selecionado, bem como as que não aceitaram participar da pesquisa.

Tabela 1 - Divisão de movelarias por Zona, dados de 2010.

\begin{tabular}{lccc}
\hline Zona & Universo (N) & $\begin{array}{c}\text { Proporção } \\
\text { (p) }\end{array}$ & Amostra (n) \\
\hline Leste & 30 & $30 / 147=0,20$ & 8 \\
Sul & 12 & $12 / 147=0,08$ & 3 \\
Norte & 40 & $40 / 147=0,27$ & 11 \\
Oeste & 20 & $20 / 147=0,14$ & 5 \\
Centro-Sul & 20 & $20 / 147=0,14$ & 6 \\
Centro-Oeste & 25 & $25 / 147=0,17$ & 7 \\
\hline Total & 147 & 1,00 & 40 \\
\hline
\end{tabular}

\subsection{Procedimentos para coleta de dados}

A coleta foi realizada por meio de entrevistas, realizadas entre fevereiro a agosto de 2013, aplcando-se formulários, e identificando-se as medidas utilizadas na confecção do mobiliário em estudo. Os dados foram obtidas por meio de visitação as movelarias indicadas pelo SEBRAE/AM e, posteriormente, foram compilados em planilha eletrônica para comparação com as recomendados pelas tabelas geradas neste estudo. O formulário para a coleta de dados foi dividido em três blocos de perguntas que, por sua vez, foram estruturados a partir de temas de interesse da pesquisa, quais sejam: Dados da empresa, buscandose obter informações para fins de identificação e caracterização da empresa; Informações sobre os produtos, identificando os produtos, seus tipos, quantidade, qualidade e forma de produção; Medição dos produtos, identificando como ocorre e quais são as utilizadas para seu dimensionamento. 
Esses blocos foram subdivididos em oito temas, quais sejam: Identificação da empresa, Caracterização da empresa, Identificação dos produtos, Quantidade dos produtos, Qualidade dos produtos, Produção, Padrão de dimensionamento e Mensuramento dos produtos. Os temas geraram 'vinte e uma' perguntas, entre abertas e fechadas, utilizando-se linguagem informal, conforme orientam Marconi e Lakatos (2011), e considerando o público ao qual o formulário foi aplicado, em sua maioria, com baixo grau de instrução (marceneiros e ajudantes). As questões geradas foram codificadas e organizadas dentro de classes a fim de facilitar sua posterior inserção em tabelas.

\subsection{Procedimentos para análise e interpretação de dados}

4.2.1. Recomendações antropométricas para dimensionamento de mobiliário proposto para a população local

Por não possuir faixas de ajustes (madeira maciça), o mobiliário foco da pesquisa contemplou as medidas recomendadas por Felisberto e Paschoarelli (2000), que possui referencia dos seguimentos corpóreos para a população brasileira, sendo a que mais se aproxima da população local, devendo-se considerar os percentis 5\% (mulher) e 95\% (homem) como principal referência dimensional, uma vez que são os mais frequentemente utilizados, como já mencionado.

Portanto, a seleção das medidas recomendadas foi realizada conforme segue:

As utilizadas como referência para o dimensionamento de cadeiras (Tabela 2) foram baseadas nas da tabela proposta por Felisberto e Paschoarelli, e se baseiam nas referencias dos seguimentos corpóreos humanos, exceto as medidas "Vão entre assento e encosto" que foram extraída de Dul e Weerdmeester (2004), "Inclinação do encosto" de Kroemer e Grandjean (2005) e "Ângulo da borda do assento" de Cakir et alii. (1978), pois tratam de medidas para dimensionamento de cadeiras.

Tabela 2 - Medidas para dimensionamento de cadeiras residenciais baseada em dados de seguimentos corpóreos humanos.

\begin{tabular}{llc}
\hline \multicolumn{2}{c}{ Medidas recomendadas para cadeiras residenciais (cm) } \\
\hline A & Altura total da cadeira & $99-$ \\
B & Almofada lombar (vão assento-encosto) & $10-20$ \\
C & Inclinação do encosto (em relação ao assento) & $105^{\circ}-110^{\circ}$ \\
D & Altura do assento & 36 \\
E & Largura do assento & 41 \\
F & Profundidade do assento & 42 \\
G & Ângulo da borda do assento & $4^{\circ}-6^{\circ}$ \\
\hline
\end{tabular}

Fonte: Adaptada de Felisberto e Paschoarelli (2000), Dul e Weerdmeester (2004), Kroemer e Grandjan (2005), Cakir et alii. (1978).

As utilizadas como referência para o dimensionamento de mesas (Tabela 3) foram extraídas de Dul e Weerdmeester (2004), por se tratarem de medidas para mesas, exceto a altura da mesa que foi extraída da tabela proposta por Felisberto e Paschoarelli (2000). Esta, pode ser obtida por meio da altura piso-joelho (do chão a parte superior do assento) mais a distância do assento até a parte debaixo do cotovelo 
flexionado (do assento até a parte superior da coxa) (QUARESMA, 2011) do homem percentil 95\%, por se tratar do usuário maior.

Tabela 3 - Medidas para dimensionamento de mesas de jantar residenciais baseada em dados de seguimentos corpóreos humanos.

\begin{tabular}{llc}
\hline \multicolumn{3}{c}{ Medidas recomendadas para mesas residenciais (cm) } \\
\hline A & Altura (h) & 72 \\
B & Largura (l) & 120 \\
C & Comprimento (c) & $91,4-$ \\
\hline
\end{tabular}

Por tartar-se de mobiliários de madeira maciça e sem possibilidade de ajustes, a única medida que poderá apresentar variação, será a de "Comprimento", uma vez que poderá ser maior de acordo com a quantidade de pessoas que deverá ser comportada à mesa. Para tanto, deve ser considerada, aquela da Limitação Lateral do Passo (Zona de Topo) que se trata da elipse corporal, vinculado à largura dos ombros $(61 \mathrm{~cm})$, baseada em uma separação interpessoal ampliada para um diâmetro de $91,4 \mathrm{~cm}$ por pessoa (HOROWITZ apud BITTENCOURT, 2011). O valor em questão proporciona maior conforto aos usuários, visto que permite sua livre movimentação.

Portanto, a dimensão 'c' (comprimento), 91,4 cm é considerada apenas para comportar uma pessoa confortavelmente, ou duas pessoas, uma de cada lado da mesa. Para comportar quatro pessoas, deve-se levar em conta que entre um indivíduo e outro (lado a lado) deve haver o espaço mínimo de 15,2 cm $(91,4 \mathrm{~cm}-61 \mathrm{~cm}=30,4 / 2=15,2 \mathrm{~cm})$. O mesmo serve para acomodação de seis ou mais pessoas.

\subsection{Procedimentos estatísticos}

Para a realização desta etapa, utilizou-se duas áreas distintas da estatística: a Descritiva e a Inferencial. Da primeira foram utilizadas as duas categorias, quais sejam:

\subsubsection{Medidas de posição}

Utilizando-se os cálculos da média, mediana, moda, máximo e mínimo, pois permitem encontrar o valor médio das dimensões da peça (altura, largura, entre outros) para a produção de móveis. O objetivo destes cálculos foi verificar os valores que se repetem com maior frequência para que possam ser posteriormente comparados aos valores recomendados por autores de ergonomia.

\subsubsection{Medidas de dispersão ou de variabilidade}

Aplicando-se cálculos de amplitude total (Equação 3), desvio-padrão (Equação 4) e coeficiente de variação (Equação 5), visto que permitem apreciar o número total de valores encontrados em uma classe e, ainda, mapear a diferenciação entre os valores obtidos.

$$
\begin{gathered}
\mathrm{A}=\text { Xmáx }- \text { Xmín } \\
\mathrm{S}=\frac{\sqrt{\sum_{\mathrm{i}=1}^{\mathrm{n}}(\mathrm{xi}-\overline{\mathrm{x}})^{2}}}{\mathrm{n}-1}
\end{gathered}
$$




$$
\mathrm{CV}=\frac{\mathrm{s}}{\overline{\mathrm{X}}} \cdot 100
$$

Como as tabelas geradas para este estudo (Tabelas 2 e 3 acima) apontam medidas de único valor, havendo também casos onde aponta intervalos, a validação dos dados procedeu por meio da comparação de médias pela aplicação do teste de $t$ de Student ao nível de $5 \%$ de probabilidade. Como o desvio padrão da população $(\sigma)$ era desconhecido, este foi estimado por meio do desvio padrão amostral $S$ e distribuição $t$ com $n-1$ de graus de liberdade, conforme afirma Costa, Cardoso Neto e Nascimento (2006).

$$
t=\frac{\bar{x}-\mu}{\frac{s}{\sqrt{n}}}(6)
$$

\subsection{Interpretação de dados}

A interpretação dos dados deu-se pela utilização de dois softwares em conjunto: Excel e SPSS. O primeiro utilizado para categorizar, organizar e visualizar os valores mensurados obtidos pela aplicação dos formulários de coleta de dados. O segundo para realização dos cálculos estatísticos pré-estabelecidos e a geração dos gráficos analíticos.

Para a validação das medidas propostas nas tabelas geradas, quanto ao dimensionamento de mesas e cadeiras em madeira maciça sem ajustes para a população local, realizou-se simulações de uso dos produtos, auxiliadas pelo utilização de dois softwares distintos: Blender, para a modelagem do mobiliário com as medidas propostas nas tabelas; e MakeHuman, destinada a modelagem dos bonecos antropométricos para testes, conforme medidas dos seguimentos corpóreos humanos proposta por Felisberto e Paschoarelli (2000).

\section{Resultados e discussão}

\subsection{Universo de pesquisa}

Os dados fornecidos pelo SEBRAE/AM (2010) apontavam um quantitativo de 147 marcenarias distribuídas pelas diversas zonas da cidade de Manaus. Durante as visitas constatou-se que, embora tivessem sido visitados $100 \%$ dos endereços indicados pelo SEBRAE/AM, apenas $41 \%$ dos estabelecimentos foram localizados, dos quais somente $36 \%$ encontravam-se em pleno funcionamento. Os $59 \%$ restantes não foram localizados ou não eram de conhecimento dos moradores locais.

Os estabelecimentos encontrados estavam distribuidos da seguinte maneira 10\% desses estabelecimentos trabalhavam com madeira maciça, 7\% apenas com MDF elou Compensado, 5\% eram madeireiras ou serralherias, $7 \%$ produziam apenas portas e janelas, 3\% eram metalúrgicas, 2\% trabalhavam apenas com revenda, outros $2 \%$ trabalhavam com outros produtos de madeira e outros $5 \%$, embora tenham sido localizados, encontravam-se desativados.

Outros $2 \%$ que também trabalhavam com móveis de madeira maciça foram encontrados por indicação dos moradores das regiões visitadas, logo, não constando na listagem.

Portanto, foi verificado in loco que a maioria das marcenarias não mais existia, uma vez que mudaram de endereço ou de negócio. Portanto, dos estabelecimentos apontados pelo SEBRAE/AM, apenas 15 
trabalhavam com produção de mobiliário em madeira maciça. Outros 4 estabelecimentos foram obtidos por meio de indicação dos moradores locais, logo, o quantitativo total de movelarias utilizadas para este estudo foi de 19 estabelecimentos.

\subsection{Análise e interpretação de dados}

Durante a verificação in loco dos 19 estabelecimentos, que trabalhavam com madeira maciça, em funcionamento, aplicou-se os formulários como forma de mapear as medidas utilizadas para a produção de mesas e cadeiras, sem ajuste, de madeira maciça, chegando-se as seguintes interpretações:

\subsubsection{Cadeiras}

Foram levantados 22 exemplares de cadeiras, bem como as dimensões de cada exemplar, estas foram comparadas com aquelas recomendadas na Tabela 2, possibilitando a aplicação dos procedimentos estatísticos já descritos. Os valores de médias e desvios padrões foram obtidos através da aplicação dos procedimentos de estatística descritiva apresentador no item Material e Métodos, possibilitando assim a aplicação do teste de $t$ de Student (Equação 6) para cada medida separadamente, conforme a seguir:

Utilizando-se da tabela de Distribuição de $t$ de Student com 21 graus de liberdade (n-1 = 22-1) e Intervalo de Confiança de $95 \%(\alpha=0,05 / 2=0,025)$, obtém-se o valor tabelado de $t(2,4138)$. Em seguida, foram calculados os valores de $t$ para as medidas Altura Total da Cadeira (A) $(t=7,44)$, Altura do Assento (D) $(t$ $=13,20)$, Largura do Assento (E) $(t=2,37)$ e Profundidade do Assento (F) $(t=0,00)$, uma vez que são as únicas que são consideradas importantes pelos marceneiros locais para a produção de cadeiras.

\section{Dimensão A - Altura total da cadeira}

Comparando-se o valor de $t$ calculado $(\mathrm{t}=7,44)$ maior do que o de $t$ tabelado $(t=2,4138)$ percebe-se que existe uma diferença significativa entre as dimensões coletadas e a dimensão recomendada pela Tabela 2 , concluindo-se, portanto que as medidas utilizadas pelos marceneiros para a altura da cadeira encontramse muito acima da recomendada, o que poderia implicar em acidentes (bater a cabeça no encosto da cadeira, quando inclinada para trás).

Dimensão D - Altura do assento

Comparando-se o valor do $t$ calculado $(t=13,20)$ com o do $t$ tabelado $(t=2,4138)$, conclui-se que as medidas de altura de assentos encontradas também apresentam diferença significativa quando comparados com a dimensão recomendada pela Tabela 2, podendo acarretar em dores musculares (compressão da poplítea, parte de trás da coxa) quando utilizadas por longos períodos de tempo.

Dimensão E-Largura do assento

Comparando-se o valor do $t$ calculado $(t=2,37)$ com o do $t$ tabelado $(t=2,4138)$, conclui-se que as medidas de largura de assentos encontradas não apresentam diferença significativa quando comparados com a dimensão recomendada pela Tabela 2, uma vez que essa dimensão se trata da largura do quadril da maior mulher. Contudo, deverão ser observados os espaços entre uma cadeira e outra, para o caso de mesas de quatro a seis lugares, respeitando-se a Zona de Topo de cada indivíduo, ou seja, entre uma cadeira e outra deverá ser respeitado o espaço de $15,2 \mathrm{~cm}$ no mínimo, conforme visto anteriormente.

\section{Dimensão F - Profundidade do assento}

Comparando-se o valor do $t$ calculado $(t=0,00)$ com o do $t$ tabelado $(t=2,4138)$, conclui-se que as medidas de comprimento / profundidade de assentos encontrados não apresentam diferenças significativas em relação a recomendada pela Tabela 2. Contudo, deve-se levar em conta que de uma amostra de 22 cadeiras analisadas, 46\% (10 exemplares) encontravam-se com o valor acima do estabelecido o que é 
considerado prejudicial, uma vez poderia causar dores musculares pelo estrangulamento da parte de trás do joelho (poplítea) o que forçaria os usuários menores a se sentarem mais próximos da ponta da cadeira, adotando posturas incorretas pela inutilização do encosto da cadeira.

Dimensões $B$ e $C$-Vão assento encosto e inclinação do encosto

Neste caso, apenas dois fabricantes apresentaram medidas consideradas padrão para o dimensionamento de seus produtos $\left(\mathrm{B}=7 \mathrm{~cm} \mathrm{e} \mathrm{C}=90^{\circ}\right)$, sendo consideradas irrelevantes ou desnecessárias para os demais.

\subsubsection{Mesas}

Foram levantados 34 exemplares de mesas sem ajustes em madeira maciça, das quais: 12 mesas de dois lugares, 17 mesas de quatro lugares e 5 a mesas de seis lugares. Foram levantadas também suas dimensões para comparações com as recomendadas na Tabela 3, possibilitando a aplicação dos procedimentos estatísticos descritos anteriormente. Os valores de média e desvio padrão, para cada uma das classes de mesas distintas, foi obtido separadamente, por meio da aplicação dos procedimentos e estatística descritiva descritos anteriormente, possibilitando a aplicação do teste $t$ de Student (Equação 6), conforme a seguir:

Utilizando-se da tabela de Distribuição de $t$ de Student com 11, 16 e 4 graus de liberdade (n-1) e Intervalo de Confiança de $95 \%(\alpha=0,05 / 2=0,025)$, obtém-se os valores tabelados de $t(t=2,5931 ; t=2,4729$ e $t$ $=3,4954)$ para os exemplares de dois, quatro e seis lugares, respectivamente. Em seguida, foram calculados os valores de $t$ para as medidas Altura da Mesa (A), Largura da Mesa (B), Comprimento da Mesa (C).

\section{Dimensão A - Altura da mesa}

Nos casos de exemplares de mesas de dois $(t=10,20)$ e quatro lugares $(t=16,87)$, foram encontradas diferenças significativas de dimensões, quando comparadas com a dimensão apontada na Tabela 3. Nos exemplares para seis lugares $(t=-3,86)$ analisados, embora apresentem a medida de $80 \mathrm{~cm}$ (todos), segundo o teste de $t$ de Student, não existem diferenças significativas. Contudo, essa dimensão quando aplicada incorretamente pode ocasionar desconforto muscular no antebraço e dores nos ombros (quando muito alta) e inclinação da coluna para frente (quando muito baixa).

\section{Dimensão B - Largura da mesa}

Para todos os casos analisados, foram encontradas variações dimensionais significativas. Por se tratar da dimensão mínima para a acomodação das pernas abaixo da mesa, essa medida é de grande importância e, levando-se em conta a configuração de duas pessoas sentadas uma de frente para a outra, a dimensão apontada na Tabela 3 deve ser respeitada, o que não ocorre.

\section{Dimensão C-Comprimento da mesa}

Para os casos de exemplares de dois $(t=12,62)$ e quatro $(t=-0,27)$ lugares foram encontradas diferenças dimensionais significativas, enquanto que nos casos de exemplares de seis lugares $(t=-2,06)$ percebeu-se que não foram encontradas variações dimensionais significativas. Contudo, para os casos de mesas de dois lugares deve-se levar em conta a medida mínima de $91,4 \mathrm{~cm}$, nas de quatro lugares $182,8 \mathrm{~cm}$ e nas de seis $274,2 \mathrm{~cm}$, ou seja, é inadmissível a utilização de medidas abaixo das dimensões indicadas, uma vez que as dimensões apontadas representam a Zona de Topo, ou seja, o espaço mínimo necessário para a acomodação de usuários, lado-a-lado, sem que um invada o espaço pessoal do outro.

Tendo em vista os dados analisados e as Tabelas 2 e 3 geradas, fez-se a simulação de uso das medidas propostas para dimensionamento do mobiliário proposto. 


\subsection{Simulação de uso}

Para fins de validação desta pesquisa, além da aplicação do teste de $t$ de Student, viu-se a necessidade de simular a aplicação das medidas recomendadas nas Tabelas 2 e 3 para a população local, ou seja, mulher percentil 5\% (menor mulher) e homem percentil 95\% (maior homem), tendo em vista que não se está projetando para um único usuário e sim para uma população inteira. Os bonecos antropométricos utilizados para as análises foram dimensionados de acordo com a tabela proposta por Fellisberto e Paschoarelli (2000) que apresenta as medidas dos seguimentos corpóreos humanos. A seguir são apresentadas as simulações de uso da cadeira com as medidas dos segmentos corpóreos humanos, sugeridas na Tabela 2, resultante deste estudo, por cada um dos percentis $(5 \%, 50 \%$ e $95 \%)$ tanto de homens (Figura 2), quanto de mulheres (Figura 3).

Observando-se as Figuras 2 e 3 nota-se que a cadeira apresentada atende desde a menor mulher (percentil $5 \%$ ) ao maior homem (percentil 95\%), uma vez que todos os usuários puderam firmar os pés no chão sem que houvesse a necessidade de sentar-se mais à frente da cadeira. Percebe-se também que, com isso, não houve o esmagamento da fossa popliteal (parte de trás do joelho), nem tampouco compressão da parte de baixo da coxa.
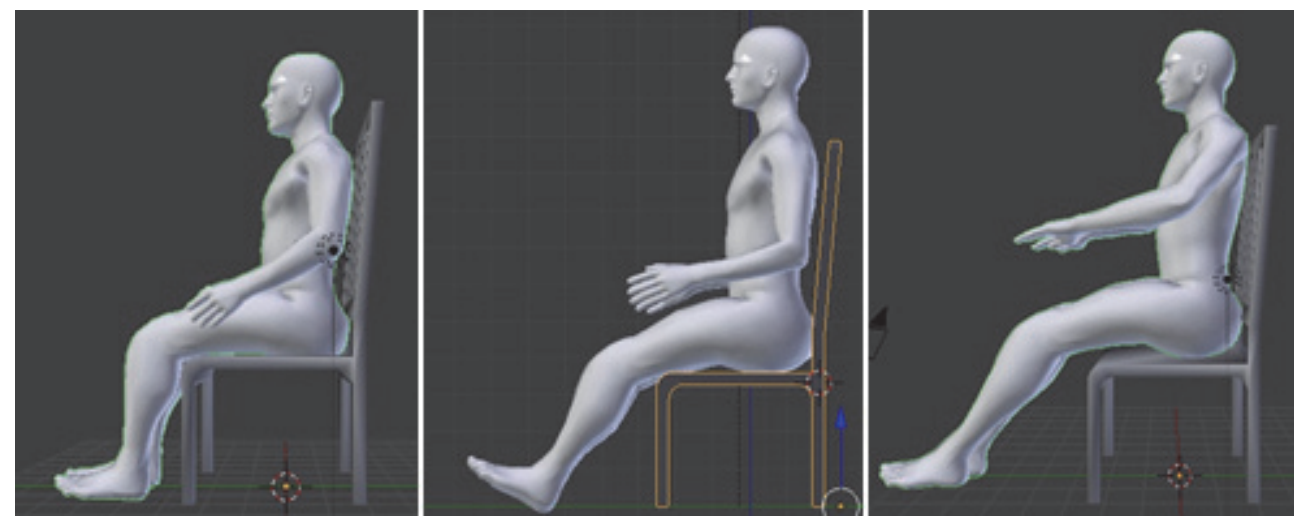

Fig. 31 - Homem percentil 5\% (menor), homem percentil 50\% (médio), homem percentil 95\% (maior), respectivamente.
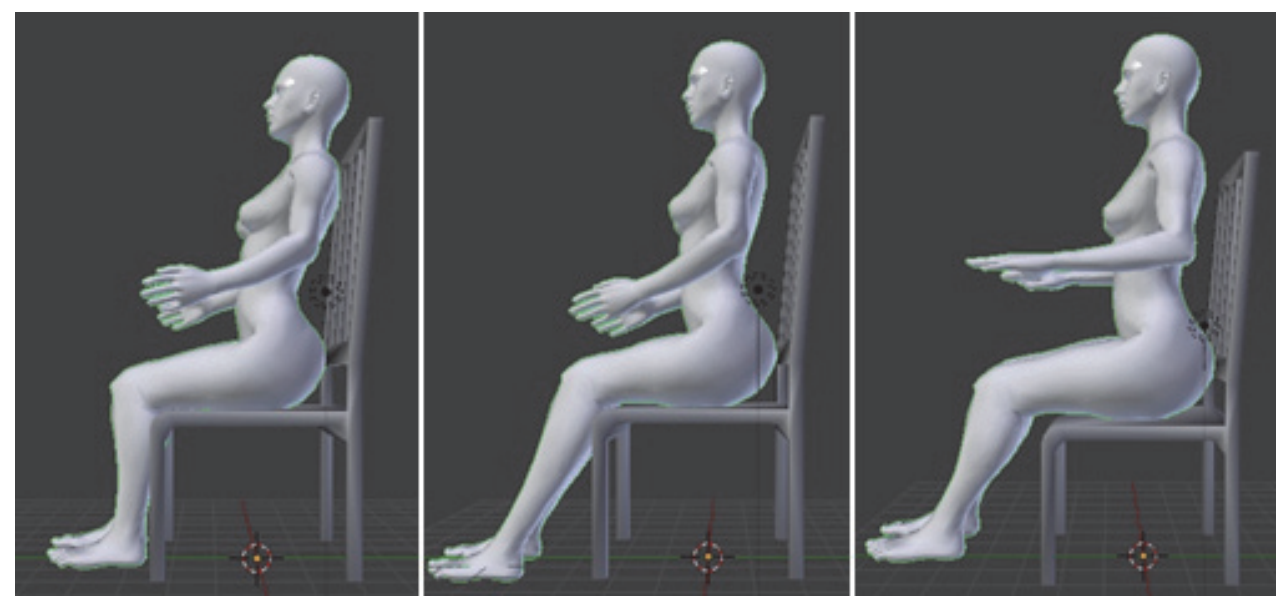

Fig. 32 Mulher percentil 5\% (menor), mulher percentil 50\% (médio), mulher percentil 95\% (maior), respectivamente. 
O mesmo tipo de simulação foi feito para as mesas (dois, quatro e seis lugares), entretanto, como todas possuem as mesmas dimensões de largura $(120 \mathrm{~cm})$ e altura $(72 \mathrm{~cm})$, utilizou-se para simular a acomodação das pernas sob e dos braços apenas um exemplo, neste caso o da mesa de dois lugares (Figura 3).

Observando-se a Figura 3 nota-se que as mesas apresentadas atendem desde a menor mulher (percentil $5 \%$ ) ao maior homem (percentil 95\%), uma vez que ambos conseguem acomodar suas pernas, sem que haja contato, conseguindo apoiar seus braços sobre o tampo da mesa sem que para isso haja a necessidade de elevar os ombros ou curvar-se para frente, adotando posturas incorretas, tensionando os músculos dos ombros e pescoço. Salienta-se que as medidas aplicadas na mesa utilizada na simulação também servem para acomodação do percentil 50\% (homens e mulheres).

Ainda sobre o caso das mesas, houve a necessidade de simular também a acomodação dos usuários ladoa-lado, para validação dos comprimentos. Para tanto, simulou-se a Zona de Topo, buscando ilustrar que as mesas apresentadas possuem espaço suficiente para a acomodação de cada indivíduo, respeitando seu espaço pessoal (Figura 4).

A Figura 4 apresenta a vista de topo da simulação de uso por homens do percentil $95 \%$, uma vez que apresentam maior largura de ombro, o que poderia dificultar sua movimentação quando acomodados próximos a outros usuários do mesmo porte. Nessa imagem, nota-se que, independentemente do tipo de mesa simulada (dois, quatro ou seis lugares), todos os usuários encontram-se perfeitamente acomodados, sem contato com os demais.

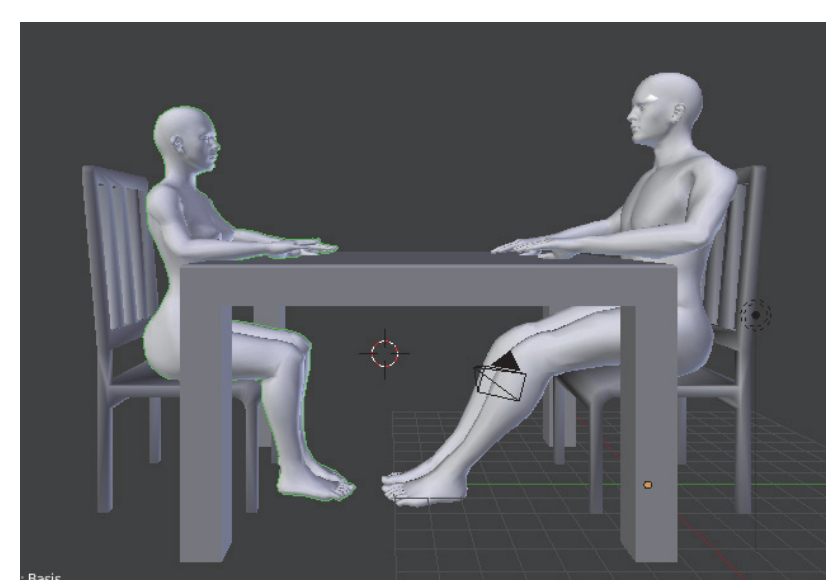

Fig. 33 À esquerda, mulher percentil 5\% (menor) e à direita, homem percentil 95\% (maior). 

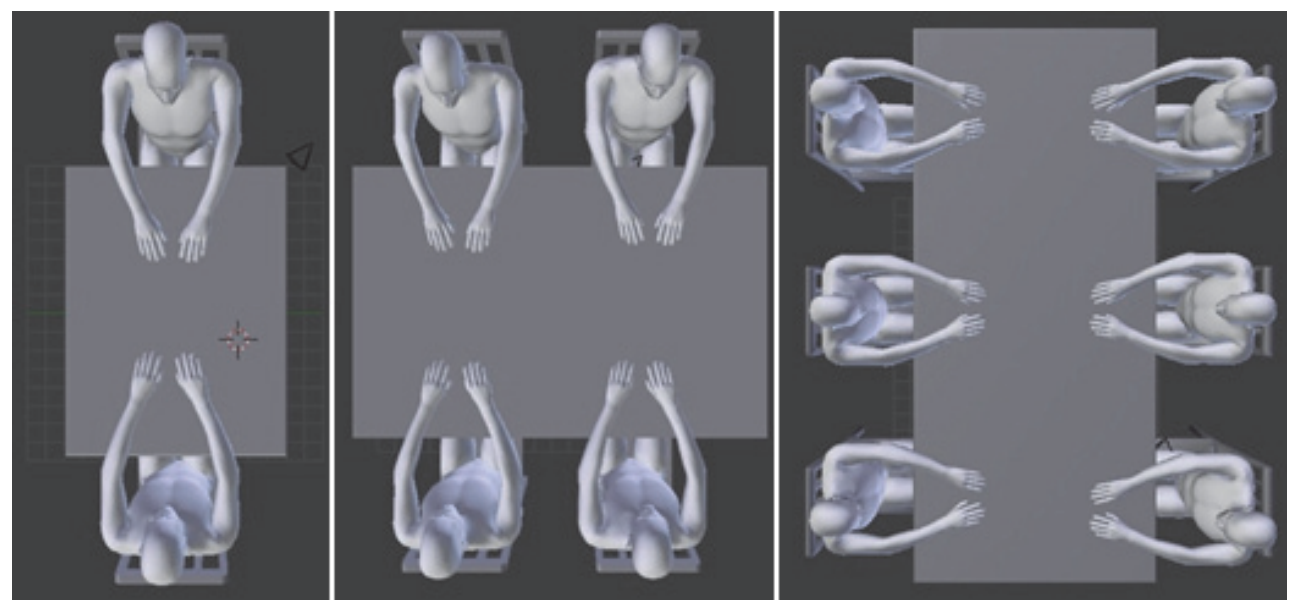

Fig 34 Acomodação dos usuários maiores (homem percentil 95\%) nas mesas de dois, quatro e seis lugares, respectivamente.

\section{Considerações finais}

Dado o exposto, percebeu-se a necessidade de disseminação das tabelas geradas neste estudo como auxilio ao correto dimensionamento de mesas e cadeiras residenciais de madeira maciça sem ajustes, produzidas e comercializadas na cidade de Manaus.

As marcenarias locais produzem e comercializam estes mobiliários de madeira maciça sem ajustes com medidas que podem ser consideradas inapropriadas, por não apresentarem padrão dimensional, sem base em normas, não somente pela falta de conhecimento técnico dos marceneiros, mas também pela ausência das mesmas, voltadas para o dimensionamento de mobiliário residencial.

Contudo, tem-se o conhecimento de uma tabela que possui medidas antropométricas dos seguimentos corpóreos humanos da população brasileira, permitindo sua adaptação para dimensionamento do mobiliário em questão, auxiliando na geração de duas novas tabelas com medidas recomendadas para dimensionar o mobiliário para a população Amazonense, tendo em vista que este estudo trata de mesas e cadeiras sem ajuste de medidas.

As tabelas geradas permitiram constatar que tanto as mesas, quanto as cadeiras residenciais de madeira maciça sem ajustes comercializadas na cidade de Manaus não são produzidas com base em recomendações ergonômicas e/ou qualquer tipo de estudo relacionado. Este argumento sustenta-se pelo seguinte: a) Cadeiras: as medidas A, D, E e F (altura total da cadeira, altura, largura e profundidade do assento, respectivamente) não se encontravam em conformidade com o recomendado por este estudo, ou outra publicação científica e as medidas B, C e G (vão assento-encosto, inclinação do encosto e ângulo da borda do assento) são ignoradas pelos marceneiros locais, embora sejam tão importantes quanto às demais; b) Mesas: as dimensões A (altura da mesa) e B (largura da mesa), consideradas críticas, necessárias para o apoio dos braços sobre a mesa, bem como para a acomodação das pernas sob a mesa, sem que haja prejuízos de saúde aos usuários, também não se encontravam em conformidade. Para ambos os tipos de mobiliário, foram encontradas medidas muito acima do indicado nas tabelas de medidas recomendadas, em alguns casos.

Portanto, entende-se que os marceneiros locais não utilizam padrão dimensional que possa ser considerado adequado ao desenvolvimento de mesas e cadeiras residenciais em madeira maciça, o que termina por tornar os seus produtos menos ergonômicos, face ao desconforto gerado pelos mesmos 
quando utilizados por longos períodos, podendo ser considerados superfaturados quando comparados com móveis industrializados, nas mesmas condições de dimensionamento, com menor preço de mercado.

Deste modo, percebe-se a necessidade da criação de mecanismos para divulgação dessas tabelas como maneira não somente de orientar, mas também de conscientizar os fabricantes acerca da importância do correto dimensionamento desses produtos por meio de apontamentos sobre o que é ergonomia e antropometria, bem como os benefícios gerados pela aplicação dessas medidas e de que forma, não só os produtores como os usuários podem ser beneficiados com isso.

\section{REFERÊNCIAS BIBLIOGRÁFICAS}

BAXTER, M. (2011) Projeto de Produto: Guia prático para o design de novos produtos. 3 ed. São Paulo: Blucher.

BITTENCOURT, F. (2011) Antropometria: Conceitos. In Fábio Bittencourt (Org.) Ergonomia e Conforto Humano: Uma visão da arquitetura, engenharia e design de interiores. 1 ed. Rio de Janeiro: Rio Books,. 196 p.;

CAKIR, A. et al., (1978) Anpassung von Bildschirmarbeitsplätzen an die physische und psychische Funktionsweise des Menschen. Bundesministerium für Arbeit und Sozialordnung. Bonn: Referat Presse.

CERVO, A. L.; BERVIAN, P. A.; DA SILVA, R. (2007) Metodologia científica. 6. ed. São Paulo: Pearson.

COSTA, S. S.; CARDOSO NETO, J.; NASCIMENTO, S. A. (2006) Metodologia quantitativa aplicada às ciências sociais. Manaus, AM: UEA.

DUL, J.; WEERDMEESTER, B. (2004) Ergonomia prática / Jan Dul, Bernard Weerdmeester; tradução Itiro Iida. 2 ed. rev. e ampl. São Paulo: Edgard Blücher.

FELISBERTO, L. C.; PASCHOARELLI, L. C. (2000) Modelos Humanos em escala para dimensionamento ergonomico preliminar de postos de trabalhos. In: Anais P\&D Design. P: 583 - 589.

KROEMER, K.H.E.; GRANDJEAN, E. (2005) Manual de Ergonomia: Adaptando o trabalho ao homem. 5. Ed. Porto Alegre: Bookman.

MARCONI, M. A.; LAKATOS, E. M. (2011) Técnicas de pesquisa, elaboração, análise e interpretação de dados. 7 ed. 5 reimpr. São Paulo: Atlas.

MÜLlER, S. I. M. G. (2012) Técnicas de pesquisa: Questionário. Disponível em: $<$ http://people.ufpr.br/ soniaisoldi/ce220/Questionarios2.pdf >. [Acesso: 23 julio 2012]

QUARESMA, M. (2011) Antropometria Aplicada. In Fábio Bittencourt (Org.) Ergonomia e Conforto Humano: Uma visão da arquitetura, engenharia e design de interiores. 1 ed. Rio de Janeiro: Rio Books.

SEBRAE - SERVIÇO BRASILEIRO DE APOIO ÀS MICRO E PEQUENAS EMPRESAS. (2010) Diagnóstico de oferta de madeira de pequenos e médios produtores florestais e da demanda atual e futura de madeira nativa elou de reflorestamento, por espécie, por parte da indústria de móveis no Estado do Amazonas. Estruturante: Madeira e Móveis da Região Amazônica. Belém: SEBRAE.

SOUZA, A. P. et al. (2010) Avaliação ergonômica de cadeiras de madeira e derivados. Revista Árvore, Viçosa, jan./fev. $<$ http://www.scielo.br/scielo.php?pid=S0100-67622010000100017\&script=sci_arttext $>$. [Acesso em: 01 out. 2010]. 